\title{
Laser-heated capillary discharge waveguides as tunable structures for laser-plasma acceleration ${ }^{\circledR P}$
}

Cite as: Phys. Plasmas 27, 093101 (2020); https://doi.org/10.1063/5.0014961

Submitted: 22 May 2020 • Accepted: 05 August 2020 • Published Online: 01 September 2020

(iD C. V. Pieronek, (D) A. J. Gonsalves, C. Benedetti, et al.

\section{COLLECTIONS}

EP This paper was selected as an Editor's Pick
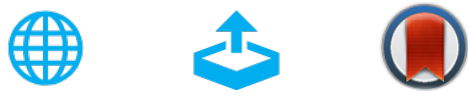

\section{ARTICLES YOU MAY BE INTERESTED IN}

Laser-heated capillary discharge plasma waveguides for electron acceleration to $8 \mathrm{GeV}$ Physics of Plasmas 27, 053102 (2020); https://doi.org/10.1063/5.0002769

Perspectives on the generation of electron beams from plasma-based accelerators and their near and long term applications

Physics of Plasmas 27, 070602 (2020); https://doi.org/10.1063/5.0004039

A compact, high resolution energy, and emittance diagnostic for electron beams using active plasma lenses

Applied Physics Letters 116, 234108 (2020); https://doi.org/10.1063/5.0005114 


\title{
Laser-heated capillary discharge waveguides as tunable structures for laser-plasma acceleration 타
}

Cite as: Phys. Plasmas 27, 093101 (2020); doi: 10.1063/5.0014961

Submitted: 22 May 2020 . Accepted: 5 August 2020 .

Published Online: 1 September 2020

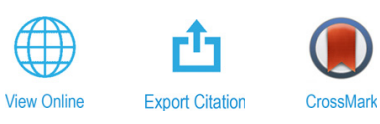

\author{
C. V. Pieronek, \\ A. J. Gonsalves, \\ C. Benedetti, ' S. S. Bulanov, ${ }^{1}$ (D) J. van Tilborg, ' J. H. Bin, \\ K. K. Swanson, \\ J. Daniels, ${ }^{1, c)}$ \\ G. A. Bagdasarov, ${ }^{4}$ N. A. Bobrova, ${ }^{4}$ V. A. Gasilov, \\ G. Korn, ${ }^{5}$ \\ P. V. Sasorov, \\ C. G. R. Geddes, \\ C. B. Schroeder, ${ }^{1,2}$ (iD W. P. Leemans, \\ and E. Esarey
}

\author{
AFFILIATIONS \\ 'Lawrence Berkeley National Laboratory, Berkeley, California 94720, USA \\ ${ }^{2}$ Department of Nuclear Engineering, University of California-Berkeley, Berkeley, California 94720, USA \\ ${ }^{3}$ Department of Physics, University of California-Berkeley, Berkeley, California 94720, USA \\ ${ }^{4}$ Keldysh Institute of Applied Mathematics RAS, Moscow 125047, Russia \\ ${ }^{5}$ Institute of Physics ASCR, v.v.i. (FZU), ELI-Beamlines Project, 18221 Prague, Czech Republic \\ ${ }^{a}$ Author to whom correspondence should be addressed: ajgonsalves@lbl.gov \\ ${ }^{b)}$ Now at: State Key Laboratory of High Field Laser Physics and CAS Center for Excellence in Ultra-intense Laser Science, Shanghai \\ Institute of Optics and Fine Mechanics, Chinese Academy of Sciences, Shanghai 201800, China. \\ ${ }^{c)}$ Now at: Deutsches Elektronen-Synchroton DESY, D-22607 Hamburg, Germany. \\ ${ }^{d)}$ Now at: Deutsches Elektronen-Synchroton DESY, D-22607 Hamburg, Germany.
}

\begin{abstract}
Published under license by AIP Publishing. https://doi.org/10.1063/5.0014961

\section{INTRODUCTION}

Laser-plasma accelerators (LPAs) have attracted intense research attention in recent years due to their ability to support extremely large accelerating gradients up to $10^{4}$ times greater than achievable in conventional accelerators. ${ }^{1}$ LPAs have numerous potential applications as compact sources of relativistic electron beams, which include freeelectron lasers and electron-positron colliders. To date, the highest energy electron beams from LPAs have been produced using preformed plasma channels, which maximize energy gain by suppressing diffraction of the driving laser pulse, enabling plasma waves to be driven at high amplitude over distances longer than the Rayleigh length. ${ }^{2,3}$ Gas-filled capillary discharge waveguides ${ }^{4}$ have been particularly successful as plasma channels for LPAs due to their simplicity of implementation and the relative ease with which they can be scaled to
\end{abstract}

Laser-heated capillary discharge waveguides are novel, low plasma density guiding structures able to guide intense laser pulses over many diffraction lengths and have recently enabled the acceleration of electrons to $7.8 \mathrm{GeV}$ by using a laser-plasma accelerator (LPA). These devices represent an improvement over conventional capillary discharge waveguides, as the channel matched spot size and plasma density can be tuned independently of the capillary radius. This has allowed the guiding of petawatt-scale pulses focused to small spot sizes within large diameter capillaries, preventing laser damage of the capillary structure. High performance channel-guided LPAs require control of matched spot size and density, which experiments and simulations reported here show can be tuned over a wide range via initial discharge and laser parameters. In this paper, measurements of the matched spot size and plasma density in laser-heated capillary discharges are presented, which are found to be in excellent agreement with simulations performed using the MHD code MARPLE. Strategies for optimizing accelerator performance are identified based on these results.

long guiding lengths. For example, $4.2 \mathrm{GeV}$ electron beams were produced using 300 TW from the BELLA laser in a $500 \mu$ m-diameter, $9 \mathrm{~cm}$-long capillary discharge operating at an on-axis plasma density $n_{e 0}$ of $7.8 \times 10^{17} \mathrm{~cm}^{-3}$.

When guiding structures are used to mitigate diffraction, beam energies are limited by depletion of the driver energy and dephasing of the electron bunch from the plasma wave accelerating field. In the quasi-linear regime, energy gain scales as $\sim a_{0} n_{e}^{-1}$, with $a_{0}$ the laser strength parameter, ${ }^{1}$ and so, the production of higher energy beams requires both increasing driver energy and reducing plasma density. Parabolic plasma channels have a plasma profile of the form $n_{e}(r)$ $=n_{e 0}+r^{2} / \pi r_{e} r_{m}^{4}$, with $r_{e}$ the classical electron radius and $r_{m}$ the matched spot size. The on-axis density $n_{e 0}$ sets the limit on energy gain due to dephasing and pump depletion, and the matched spot size 
$r_{m}$ characterizes the strength of the guide. If a low power Gaussian beam is coupled into a channel at focus with $r_{m}$ equal to its beam waist $r_{0}$, the guided beam will maintain a constant size, while it propagates in the channel. If the beam waist and matched spot size are not equal, however, the guided beam will undergo oscillations in spot size as it propagates, degrading accelerator performance and possibly destroying the guiding structure. In the quasi-linear regime, self-focusing helps confine laser energy near the axis and relaxes the condition for matched propagation, but this amounts to a modest increase in the required matched spot size and does not eliminate the need for a preformed plasma channel to guide over long distances.

The density profile of a capillary discharge waveguide is closely approximated by a parabola near the axis, and the matched spot size obeys the scaling $r_{m} \propto r_{c}^{1 / 2} n_{e 0}^{-1 / 4}$, with $r_{c}$ the capillary radius. This scaling law dictates that the only means of tuning $r_{m}$ and $n_{e 0}$ in a capillary discharge waveguide are the fill gas pressure and the capillary radius, with the latter fixed at the time of fabrication. The matched spot size $r_{m}$, then, becomes a strictly decreasing function of $n_{e 0}$, leaving a capillary discharge waveguide with effectively one degree of freedom.

This coupling of $r_{m}$ and $n_{e 0}$ is problematic for efforts to increase the performance of channel-guided LPAs. First, reducing $n_{e 0}$ to increase energy gain requires reducing the capillary radius to avoid increasing $r_{m}$ and weakening the channel, which, in turn, exposes the capillary to laser damage from the wings of the driver beam. For example, reducing plasma density from $7.8 \times 10^{17} \mathrm{~cm}^{-3}$, which produced $4.2 \mathrm{GeV}$ with a power of $300 \mathrm{TW}$ from the BELLA laser, to $2.7 \times 10^{17} \mathrm{~cm}^{-3}$, which produced $7.8 \mathrm{GeV}$ with a laser power of 880 TW, would have required a $40 \%$ decrease in the capillary radius in order to maintain the same matched spot size. However, the previous experiments on the BELLA laser with $500 \mu \mathrm{m}$ diameter capillaries operated at $n_{e 0}=7.8 \times 10^{17} \mathrm{~cm}^{-3}$ observed destruction of the capillary by energy in the wings of the focal spot when laser power was increased above $300 \mathrm{TW}$. Therefore, capillaries of that size or smaller cannot be used at petawatt-scale laser power, even with state-of-theart laser modes.

Additionally, independent control of $r_{m}$ and $n_{e 0}$ is useful for tuning of a laser-plasma accelerator: $r_{m}$ can be optimized for matched guiding, and $n_{e 0}$ can be optimized for acceleration, e.g., pump depletion and bunch dephasing or bunch injection. Furthermore, at high laser power, the channel matched spot size, on-axis plasma density, plasma wave phase velocity, and laser intensity are all coupled. ${ }^{6}$ Independent control of $n_{e 0}$ and $r_{m}$ adds a degree of freedom, which may be useful for optimization under this coupling. For example, an injection scheme may place constraints on laser intensity and plasma density, requiring an independent adjustment of matched spot size to preserve guiding.

In order to meet the requirements on $r_{m}$ and $n_{e 0}$ for high energy electron beam production without exposing the capillary to laser damage and independently tune $r_{m}$ and $n_{e 0}$ to optimize accelerator performance, greater control of the plasma profile beyond that afforded by a conventional gas-filled capillary discharge is needed. To provide this additional control of the plasma profile independent of the capillary radius, the laser-heated capillary discharge was developed. ${ }^{8-10}$ In this scheme, a joule-level, nanosecond-scale laser pulse is coupled into a capillary discharge waveguide, which heats the center of the plasma through inverse-bremsstrahlung absorption. The expansion of the heated plasma reduces the density on-axis, creating a channel with a smaller matched spot size than the capillary discharge alone. ${ }^{8}$ This new structure recently enabled the production of electron beams with energies up to $7.8 \mathrm{GeV}$.

In this paper, a detailed characterization of laser-heated capillary discharge waveguides is presented, relying on experiments and on simulations with the magnetohydrodynamics code MARPLE. ${ }^{11}$ Trends are identified in channel properties relevant to optimizing laserplasma acceleration with respect to plasma density and temperature and laser energy. Relatedly, the matched spot-size and on-axis density are found to be independently tunable over a large parameter space. In general, agreement between experimental measurements and MARPLE simulations is excellent.

In addition to the tuning of channel properties, several new results are presented, which are relevant to the design and operation of laser-plasma accelerators implementing laser-heated capillary discharges. Channel properties are found to be very sensitive to delay relative to the heater pulse due to rapid evolution of the plasma, enabling further optimization of laser-guiding and accelerator performance over previous experiments, which were performed with the driver injected only at the peak of the heater pulse. ${ }^{9,10}$ Also, the effect of plasma heating was observed to saturate with heater pulse energy, which is shown to be a consequence of the temperature dependence of inverse-bremsstrahlung absorption. Finally, the heater beam and hence plasma channel were steered away from the capillary axis for sufficiently high heater pulse energies, which was attributed to the transverse asymmetry of the beam.

This paper is organized as follows: In Sec. II, channel formation by inverse-bremsstrahlung heating is discussed. In Sec. III, the experiment setup and methods are described. The results of experimental measurements and simulations of the formation of a plasma channel by laser heating are presented in Sec. IV, and tuning of channel properties through laser and plasma parameters is demonstrated in Sec. V. Conclusions are summarized in Sec. VI. Appendixes A and B discuss the matched spot size diagnostic and experimental uncertainties in the density measurements in greater detail, respectively.

\section{PLASMA RESPONSE TO INVERSE- BREMSSTRAHLUNG HEATING}

In a laser-heated capillary discharge, a channel is formed by the expansion of plasma near the discharge axis upon heating by an energetic laser pulse propagating collinearly with the capillary axis. Similar to other types of laser-formed or laser-enhanced plasma channels, heating and expansion of the plasma on axis enhance the channel by lowering on-axis density and the matched spot size. ${ }^{12,13}$ In the regime under consideration, heating occurs through inverse-bremsstrahlung absorption, ${ }^{14-16}$ which is described by the imaginary part of the complex permittivity $\epsilon=\epsilon^{\prime}+i \epsilon^{\prime \prime}$. For the relatively low laser intensities $\left(\sim 10^{12} \mathrm{~W} / \mathrm{cm}^{2}\right)$ used in the experiments described here, the inversebremsstrahlung power density in the Kramers approximation is given by $^{14}$

$$
Q_{I B}=k \epsilon^{\prime \prime} I=\frac{2^{5 / 2} \pi^{1 / 2}}{3} \frac{Z q_{e}^{4} n_{e} I}{\left(k_{b} T_{e}\right)^{3 / 2} m_{e}^{1 / 2} c} \frac{\omega_{p}^{2}}{\omega^{2}} \Lambda,
$$

with $\omega, k$, and $I$ the laser frequency, wavenumber, and intensity, $q_{e}$ and $m_{e}$ the electron charge and mass, $Z$ the ionization degree, $n_{e}$ the electron density, $\omega_{p}$ the electron plasma frequency, $T_{e}$ the electron temperature, $k_{b}$ the Boltzmann constant, and the Coulomb logarithm 


$$
\Lambda=\max \left\{\frac{\pi}{\sqrt{3}}, \frac{1}{2} \ln \frac{2^{5}\left(k_{b} T_{e}\right)^{3}}{\exp \left(5 C_{\gamma}\right) \omega^{2} Z^{2} q_{e}^{4} m_{e}}\right\},
$$

where $C_{\gamma}=0.57721 \ldots$ is the Euler-Mascheroni constant. Equation (1) is valid when $T_{e} \ll Z^{2} q_{e}^{4} m_{e} / \hbar^{2} \sim 27 Z^{2} \mathrm{eV}$ and $\omega \gg \omega_{p}$.

The inverse-bremsstrahlung power density [Eq. (1)] was implemented in the MARPLE magnetohydrodynamics code, the relevant equations of which are described in Refs. 7 and 17-19. Laser absorption by the plasma first proceeds through electron heating via inversebremsstrahlung, followed by collisional heat transfer to the ions. Plasma expansion is driven by the resulting increase in pressure and temperature of both the electrons and ions. Generally, $T_{e}>T_{i}$ if plasma heating occurs on a timescale shorter than the characteristic timescale for electron-ion energy exchange. However, this timescale is of order 100 ps for the plasmas typical of the experiments reported here, which have temperatures $T_{e}$ of a few $\mathrm{eV}$ and initial densities $n_{e}$ of a few $10^{17} \mathrm{~cm}^{-3}$. Hence, for the plasmas under consideration, the approximation $T_{e} \approx T_{i}$ can be made for inverse-bremsstrahlung heating with few-ns laser pulses.

Inverse-bremsstrahlung absorption is a collisional process. Using Eq. (1) and approximating $T_{e} \approx T_{i}$, a simplified model of the plasma temperature evolution can be written in terms of the electron ponderomotive energy $E_{p}=2 \pi q_{e}^{2} I / m c \omega^{2}$ and an effective collision frequency $\nu_{e, I B}{ }^{7,17}$

$$
\frac{\mathrm{d}\left(k_{b} T_{e}\right)}{\mathrm{d} t}=\frac{\mathrm{d}\left(k_{b} T_{i}\right)}{\mathrm{d} t}=\frac{1}{2} \nu_{e, I B} E_{p}
$$

with

$$
\nu_{e, I B}=\frac{2^{7 / 2} \pi^{1 / 2}}{3} \frac{Z q_{e}^{4} n_{e}}{m_{e}^{1 / 2}\left(k_{b} T_{e}\right)^{3 / 2}} \Lambda
$$

from rearranging the factors of Eq. (1) and the factor $1 / 2$ from the partition of the absorbed energy between electrons and ions. The experiments described here involve peak laser intensities up to $4 \times 10^{11} \mathrm{~W} / \mathrm{cm}^{2}$, yielding a heating rate of a few $\mathrm{eV} / \mathrm{ns}$ for the plasma parameters stated previously.

Equation (3) describes the heating of electrons and ions by the extraction of electron ponderomotive energy at the effective collision frequency Eq. (4), which is closely analogous to resistive heating and obeys a very similar scaling law,

$$
\frac{\mathrm{d} T_{e}}{\mathrm{~d} t} \propto \frac{n_{e} I}{\omega^{2} T_{e}^{3 / 2}} \Lambda
$$

It is apparent from this relation that the heating rate increases with plasma density and laser intensity and decreases with temperature. As will be shown later, the dependence of the heating rate on these variables enables tuning of channel properties in a laser-heated capillary discharge.

The effect of plasma motion in response to heating on the propagation of the heater beam is an important consideration in the formation of a channel by laser heating. The effect becomes important if the laser pulse length is similar to the timescale for plasma motion, which can be estimated with the ion acoustic speed $c_{s}=\sqrt{\left(k_{b} T_{e}+k_{b} T_{i}\right) / m_{i}}$, where $T_{i}$ and $m_{i}$ are the ion temperature and mass, respectively. For plasma parameters representative of these experiments, and taking the spatial scale length $L$ equal to the heater laser beam waist $r_{0, h}=82 \mu \mathrm{m}$, the timescale for plasma motion is $\sim 3 \mathrm{~ns}$.
The first simulations of laser-heated capillary discharges considered a $1 \mathrm{~ns}$ pulse length, which was chosen to be shorter than the plasma motion timescale. ${ }^{8}$ In this regime, the heater beam propagation is mostly decoupled from plasma motion because the plasma is nearly stationary during the pulse, and the pulse propagates as in a linear medium. However, this also requires that the heater be matched to the initial discharge channel to avoid oscillations and nonuniform energy deposition. This limits the heater spot size to the matched spot size of the initial discharge channel, which, for the parameters of interest, is quite large, and thus limits the matched spot size of the heated channel. In Ref. 8, the heater beam reduced the matched spot size of the channel from 145 to $100 \mu \mathrm{m}$. This is much larger than the $\sim 60 \mu \mathrm{m}$ focal spot of the BELLA laser and insufficient for good confinement of driver energy toward the axis, even accounting for self-focusing effects.

It can be advantageous to use heater pulses significantly longer than the few-nanosecond plasma motion timescale. In this regime, plasma expansion during heating forms a channel during the rise of the pulse, and therefore, much of the energy is guided with a smaller effective matched spot size than the discharge alone. This "selfchanneling" effect relaxes the requirement to match the heater beam spot to the initial discharge channel, enabling the production of smaller matched spot sizes than in the short pulse regime. ${ }^{9,10}$ To take advantage of this effect, an 8 ns heater pulse length was used in the electron beam production experiments in Refs. 9 and 10 and the experiments reported here.

\section{EXPERIMENTAL SETUP AND METHODS}

The setup used for the experiments described in this paper is pictured schematically in Fig. 1(a). The capillary and heater laser system were selected to match the parameters of the electron acceleration experiments of Refs. 9 and 10. A $800 \mu \mathrm{m}$-diameter, $20 \mathrm{~cm}$-long hydrogen-filled capillary was used, driven by a high voltage pulser generating current pulses of the form shown in Fig. 1(b), which peak at $450 \mathrm{~A}$ with a FWHM of $500 \mathrm{~ns}$. A frequency doubled Nd:YAG laser system was used for laser heating, supplying up to $300 \mathrm{~mJ}$ at $532 \mathrm{~nm}$ in an $8 \mathrm{~ns}$ FWHM pulse, which was focused to a $1 / e^{2}$ spot size of $82 \mu \mathrm{m}$. The heater laser beam was non-Gaussian and significantly asymmetric in the midfield, which is evident in the beam profiles shown in Fig. 2. A separate probe laser was used to diagnose channel properties. It consisted of $4 \mathrm{~nJ}$ sub-ns pulses at $780 \mathrm{~nm}$ with a bandwidth of $30 \mathrm{~nm}$ carried by a single mode fiber, which were injected collinearly with the heater laser using a fiber collimator and Galilean telescope. The probe beam was near Gaussian at focus, with a $73 \mu \mathrm{m}$ waist and beam quality factor $M^{2}=\pi \theta_{0} r_{0} / 2 \lambda \lesssim 1.3$, with $\theta_{0}$ the divergence angle and $\lambda$ the laser wavelength $\left(M^{2}=1\right.$ for a pure Gaussian TEM $\mathrm{T}_{00}$ beam).

Separate CCD cameras for each laser wavelength were used for beam tuning and imaging the transmitted laser beam at the capillary exit. For accurate measurements, it is essential to align the probe precisely to the laser-heated channel. This was done by first aligning the probe and heater beams to each other in vacuum and then aligning the capillary to the heater beam. To facilitate this, the probe imaging line consisted of an achromatic doublet in the chamber after the capillary and a second doublet before the stage mounted microscope lens and camera, and was capable of imaging the heater beam with an appropriate change of filtration. This system was used to overlap the probe and heater beam to within $\leq 20 \mu \mathrm{m}$ in position and $\leq 100 \mu \mathrm{rad}$ in angle. 
(a)

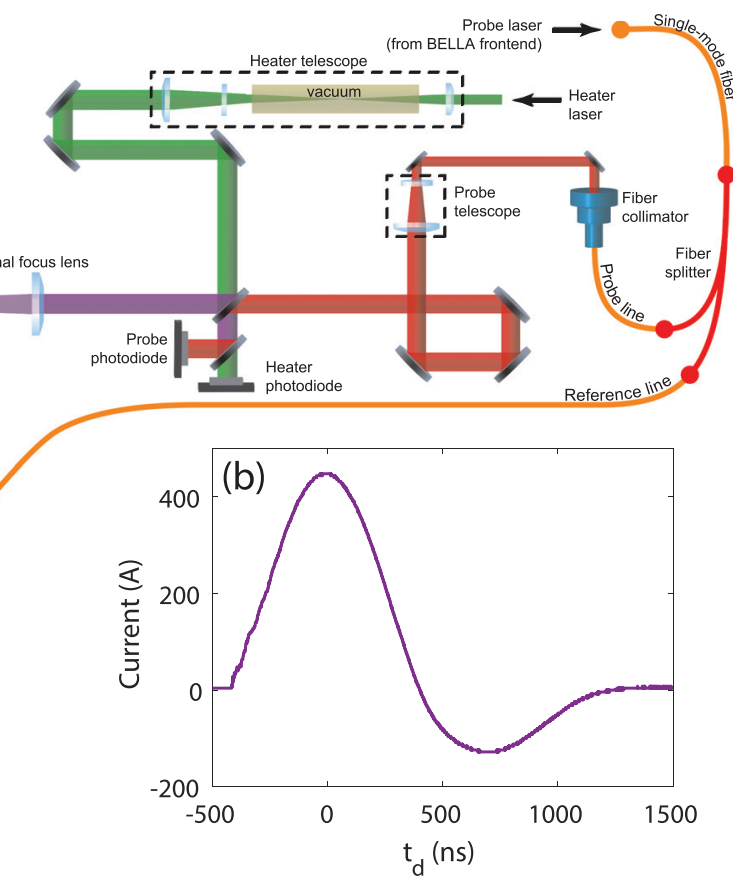

FIG. 1. (a) Schematic of the optical setup for performing density and matched spot size measurements of laser-heated capillary discharges. (b) Discharge current pulse.

The relative delay of the probe and heater pulses, as well as the heater pulse shape, was recorded for every shot with a pair of photodiodes upstream of the target. Leakage light from the final steering mirror was separated into both wavelengths with a dichroic optic, which were then sent to the photodiodes. The timing jitter between the two pulses was $\sim 0.3 \mathrm{~ns}$.

The longitudinally averaged plasma density on the channel axis $\bar{n}_{e 0}$ was diagnosed by measuring the average group velocity of the guided probe pulses using spectral interferometry. ${ }^{20,21}$ The group velocity of a plane electromagnetic wave propagating in a homogeneous plasma is $\beta_{g}=v_{g} / c \simeq 1-\beta_{n_{e 0}}$, with $\beta_{n_{e 0}}=k_{p}^{2} / 2 k^{2}$ and $k_{p}$ and $k$ the plasma and laser wavenumbers. The small term $\beta_{n_{e 0}}$ is proportional to plasma density and manifests as an additional delay of the probe pulse compared to propagation through vacuum. Neglecting the transverse variation of the plasma profile, for a $20 \mathrm{~cm}$ capillary with an on-axis density of $3 \times 10^{17} \mathrm{~cm}^{-3}$, this yields an additional group delay $\Delta T \approx 60$ fs relative to vacuum.

A fiber-based spectral interferometer was built for performing the group velocity measurements. A 50-50 fiber splitter upstream of the fiber collimator splits the probe pulses, sending one copy through the target and another to the reference arm of the interferometer. A movable dichroic optic was positioned inside the target chamber after the collimating doublet that can be moved into the beam line to send probe pulses to the interferometer for group velocity measurements. Although the spectrometer does not image the capillary exit, the collimating doublet and the lens before the slit render the delay measurement insensitive to displacement and angular deflection of the probe beam in the capillary exit plane. Ray tracing simulations showed that these effects contribute an uncertainty $\lesssim 1 \mathrm{fs}$ or $\leqq 5 \times 10^{15} \mathrm{~cm}^{-3}$ in these experiments.

The most important source of random error in the density measurement is jitter in the relative delay of the probe and reference pulses from the vibration of optics in the setup. Optical fibers were used in the interferometer to reduce this delay jitter, at the cost of introducing a relative spectral chirp between the probe and reference pulses and a temperature drift in the measured group delay, due to the dispersion and temperature dependent refractive index of the fibers, respectively. These issues were addressed through the use of an FFT-based phase

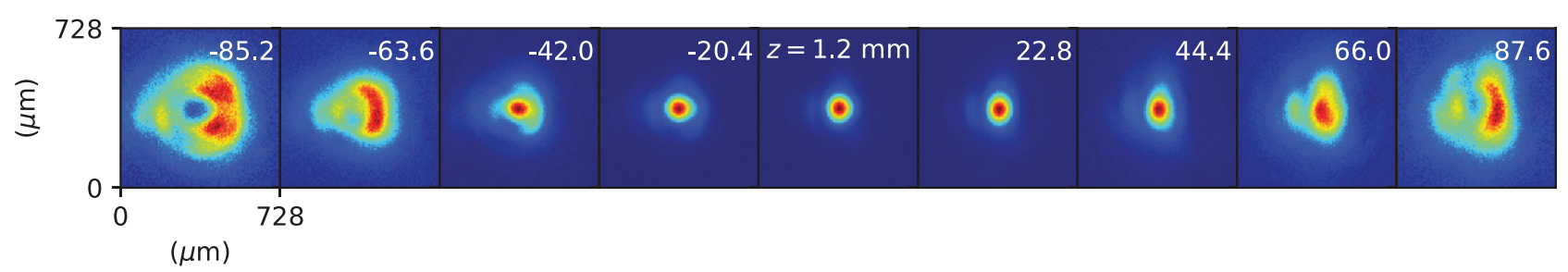

FIG. 2. Heater beam profile as a function of distance $z$ from the capillary entrance plane. Images are plotted with a normalized scale to show details away from focus. 
mapping technique ${ }^{22}$ and a data acquisition procedure that tracked the group delay drift during measurements.

The matched spot size was diagnosed using well-known techniques based on laser propagation in plasma channels. The spot size of a Gaussian laser beam injected into a mismatched parabolic channel will oscillate with wavenumber $4 / \mathrm{kr}_{m}^{2}{ }^{2}{ }^{23,24}$ This oscillation in laser spot size is a diagnostic for $r_{m}$ and particularly well suited for laser-heated capillary discharges. If $r_{m}$ is known for some set of plasma parameter values, oscillations in exit beam size, which occur as plasma parameters are slowly varied away from those values, can be counted to determine $r_{m}$ for a different set of parameter values. For laser heated channels, MARPLE calculations show significant longitudinal variations in the plasma profile. Therefore, in these experiments, spot size oscillation tracking yields the longitudinally averaged matched spot size $\bar{r}_{m}$. Because the probe beam was non-Gaussian, the INF\&RNO $\operatorname{code}^{25,26}$ coupled with a detailed phase and amplitude model of the probe laser was used to map oscillation periods to $\bar{r}_{m}$ values (see Appendix A).

Similar to spot-size oscillation, the centroid of a laser beam injected into a plasma channel off-axis will oscillate within the channel with a wavenumber $k_{\beta c}=2 / k_{m}^{2}$. ${ }^{23,24}$ Laser centroid oscillation is an accurate and easily implemented diagnostic for capillary discharges, ${ }^{24}$ but it is more difficult to implement for laser-heated capillary discharges because the measurements require precisely displacing the channel from the probe beam. The difficulty arises from pointing jitter of the heater beam, which introduces an uncertainty in the position of the channel and increases the measurement error. Spot size oscillations, however, are relatively insensitive to alignment of the probe beam to the channel. Therefore, $\bar{r}_{m}$ in laser-heated channels was measured by using centroid oscillations to determine $r_{m}$ for the capillary discharge in the absence of heating (in which case $r_{m}=\bar{r}_{m}$ ) and then using spot size oscillations to track the change in $\bar{r}_{m}$ as heating was gradually increased to the value of interest for the measurement (see Appendix A).

Accurate density measurements using the channel group velocity require knowledge of $r_{m}$ and the true propagation distance within the channel $L$. The group velocity in a parabolic channel is lower than in a homogeneous medium, possessing a term $\bar{\beta}_{g, g e o} \propto r_{m}^{-2}$ as described in Ref. 27. In these experiments, this reduction in group velocity increases the apparent on-axis density by a maximum of $4 \times 10^{16} \mathrm{~cm}^{-3}$ at $\bar{r}_{m}=60 \mu \mathrm{m}$.

Additionally, it was found that the transverse asymmetry of the heater laser mode resulted in the creation of curved plasma channels. As discussed later, this is evidenced by an intensity dependent deflection of the heater beam as it exits the capillary. It is, thus, inferred that the center of the heated channel does not remain in the center of the capillary and in fact follows a curved path through the capillary. This occurs because the tail of the heater pulse is "steered" by the channel created by the head of the pulse in a similar manner to selfchanneling, where the tail of the heater pulse is guided in the channel created by the head as described in Refs. 9 and 10. This "channel curvature" causes the probe beam to become misaligned as it propagates and undergo centroid oscillation, introducing additional path length $\delta L \propto \bar{r}_{m}^{-2}$ and increasing apparent plasma density. The degree of channel curvature is dependent on the properties of the heater beam, and the effect was not observed in the experiments of Refs. 9 and 10, which used a heater beam with a more symmetric mode. For the experiments in this paper, it is estimated that this effect contributes a maximum of $4 \times 10^{16} \mathrm{~cm}^{-3}$ to the apparent on-axis density at $\bar{r}_{m}=60 \mu \mathrm{m}$.

Precisely modeling the group velocity reduction from finite matched spot size and the increased path length from channel curvature is difficult and lies beyond the scope of this paper. Therefore, these propagation effects are estimated here using a model of a misaligned probe beam propagating in a longitudinally uniform parabolic channel (see Appendix B). Because of the limitations of this simplified model, these effects are treated as a systematic error defining the lower uncertainty bound on the plasma density.

\section{CHANNEL FORMATION AND EVOLUTION IN A LASER-HEATED CAPILLARY DISCHARGE}

Experiments and MARPLE simulations show significant modification of the plasma density by laser heating, with reductions of both $\bar{n}_{e 0}$ and $\bar{r}_{m}$. Figure 3 shows a case for a heater pulse energy of $300 \mathrm{~mJ}$, arriving at the capillary entrance at a delay $t_{d}$ of $440 \mathrm{~ns}$ after the peak of discharge current, with initial on-axis plasma density $n_{e 0, i}=3.9$ $\times 10^{17} \mathrm{~cm}^{-3}$. Images of the guided probe beam are shown in Fig. 3(a) for a range of delays $t$ relative to the centroid of the heater pulse. In

(a)
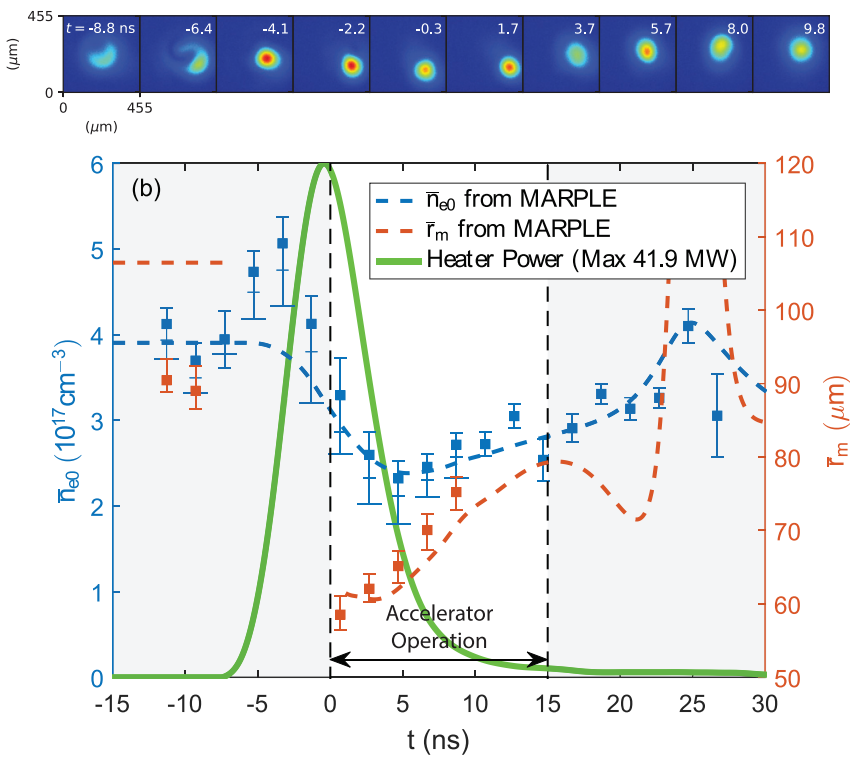

FIG. 3. Guided laser beam and channel properties for a $20 \mathrm{~cm}$-long, $800 \mu \mathrm{m}$-diameter capillary filled with 17.6 Torr of $\mathrm{H}_{2}$ and heated with a laser pulse energy of 300 $\mathrm{mJ}$. (a) Images of the guided probe beam at the capillary exit vs delay from the peak of the heater pulse. Jitter in the probe beam position at the capillary exit was $<10 \mu \mathrm{m}$. (b) Longitudinally averaged on-axis density $\bar{n}_{\mathrm{e} 0}$ and longitudinally averaged matched spot size $\bar{r}_{m}$ vs delay $t$ from the heater pulse centroid from measurements and a MARPLE simulation. The representative heater power trace used in the MARPLE calculations (obtained from averaged photodiode traces) is shown in green. Narrow-cap error bars denote uncertainty from group delay jitter between the probe and reference pulses. Wide-cap error bars denote uncertainty in the density measurement from channel curvature and finite matched spot size effects. $\bar{n}_{e 0}$ and $\bar{r}_{m}$ values are omitted when the MARPLE simulation shows that the channel size is below the input laser spot size of $73 \mu \mathrm{m}$. Driver arrival times typical for accelerator operation are marked, with shading applied for times outside this interval. 
Fig. 3(b), $\bar{n}_{e 0}$ and $\bar{r}_{m}$ from measurements and a MARPLE simulation, and the heater power, are shown as a function of delay $t$. The heater power trace shown was obtained by averaging 1000 photodiode traces and smoothing with a lowess fit and was used to model the heater pulse in the MARPLE simulations.

An average on-axis density of $2.4 \times 10^{17} \mathrm{~cm}^{-3}$ and a matched spot size of $65 \mu \mathrm{m}$ are measured at $t=4.6 \mathrm{~ns}$, parameters suitable for acceleration to energies approaching $10 \mathrm{GeV} .^{10,28}$ Because of the scaling $r_{m} \propto r_{c}^{1 / 2} n_{e 0}^{-1 / 4}$, realizing these parameters in a capillary discharge would require a $60 \%$ reduction in the capillary diameter from $800 \mu \mathrm{m}$ to $\sim 300 \mu \mathrm{m}$. Capillaries of this size are vulnerable to damage from the BELLA laser and are unsuitable for petawatt-scale experiments, based on the destruction of $500 \mu$ m-diameter capillaries observed above 300 TW of laser power in the experiments of Ref. 5 .

Modification of the guided probe beam can be seen in Fig. 3(a) as early as $t=-6.4 \mathrm{~ns}$, indicating significant evolution of the plasma profile $\sim 1 \mathrm{~ns}$ after the foot of the heater pulse at $t=-7.4 \mathrm{~ns}$. This is consistent with the $3 \mathrm{~ns}$ timescale for plasma motion calculated in Sec. II. The modification of the guided probe beam beginning at $t=-6.4 \mathrm{~ns}$ in Fig. 3(a) is also evidence of self-channeling of the heater beam. The evolution of the guided probe beam early in the heater pulse shows that the majority of the pulse energy propagates through a plasma channel with different guiding properties than the discharge before heating at $t=-8.8 \mathrm{~ns}$. It must be noted that an increase in probe intensity in the exit plane does not necessarily correspond to a reduction in matched spot size. Rather, this indicates that a minimum in the probe spot size oscillation has moved near the exit plane, which can occur during either an increase or a decrease in $\bar{r}_{m}$ depending on the oscillation phase.

The data of Fig. 3 show that the channel evolves on a nanosecond timescale and that properly timing the driver pulse relative to the heater pulse is critical for achieving maximum accelerator performance. In typical operation, the driver pulse is timed to arrive during the time interval extending roughly from $t=0 \mathrm{~ns}$ to $15 \mathrm{~ns}$ after the peak of the heater pulse, as density and matched spot size are significantly reduced from the discharge values during this time. This interval is marked in Fig. 3, with times lying outside it shaded. For the experiments of Refs. 9 and 10, the driver was timed at $t \sim 1 \mathrm{~ns}$. However, the data of Fig. 3 show that $\sim 5 \mathrm{~ns}$ after the peak of the heater pulse, the plasma density reaches a minimum with $r_{m} \lesssim 65 \mu \mathrm{m}$, still sufficiently well matched to the $\sim 60 \mu \mathrm{m}$ BELLA beam waist. Hence, it is expected that accelerator performance can be improved over previous experiments by taking full advantage of the plasma evolution to achieve optimal channel properties.

\section{A. MARPLE simulation}

For the simulations in this paper, the MARPLE code was augmented with modules from INF\&RNO and implemented the inversebremsstrahlung power density [Eq. (1)]. The heater beam intensity at the capillary entrance was modeled as a function of the form $J_{1}^{2}(r) / r^{2}$ with a $1 / e^{2}$ radius of $82 \mu \mathrm{m}$, where $J_{1}(r)$ is a Bessel function of the first kind, and the laser power was modeled using the trace in Fig. 3. The simulations were performed on an $800 \mu$ m-diameter, $20 \mathrm{~cm}$-long, axisymmetric, uniform $(r, z)$ grid with 50 cells in the radial direction and 200 cells in the longitudinal direction, with a time step of $0.1 \mathrm{~ns}$. Plasma evolution was simulated from the initiation of the discharge $420 \mathrm{~ns}$ before the peak of current, through the arrival of the heater laser pulse, to $1000 \mathrm{~ns}$ after the peak of current. The initial neutral hydrogen density was chosen to match the measured density at the arrival time of the heater pulse. The discharge plasma was modeled as initially longitudinally uniform, ignoring the $6 \mathrm{~mm}$ density ramp at each end of the capillary arising from gas flow out the ends into the surrounding vacuum. These "end effects" are expected to have little effect on guiding since the $6 \mathrm{~mm}$ length of the density ramp is smaller than the 20 and $40 \mathrm{~mm}$ Rayleigh lengths of the probe and heater beams, respectively.

The longitudinally averaged matched spot size $\bar{r}_{m}$ was calculated from the MARPLE output by computing the quasi-matched Gaussian spot size derived in Ref. 29 for each longitudinal grid point of the calculated electron density $n_{e}(r, z)$ and then averaging in $z$. This definition of the matched spot size was used because it is well defined for non-parabolic plasma profiles. MARPLE simulations show that early in the heater pulse, the density well formed by laser heating is small in radial extent because the heated plasma has had little time to expand under its own pressure. In this situation, the channel may be smaller than the guided beam, and a large fraction of the beam will not be coupled into the channel formed by heating. Instead, the beam will effectively sample two guiding structures: both the density well on axis from heating and the density well formed by the background plasma profile of the discharge. Another condition that may arise is the formation of a local maximum of the plasma density on the channel axis, due to plasma motion driven by uneven heating in the transverse plane by the mismatched, multimode heater beam.

Both of these conditions constitute a qualitative departure from the model on which the spot size oscillation diagnostic is based, which assumes propagation in a single, parabolic density well and thus renders the measurement difficult to interpret. Therefore, $\bar{r}_{m}$ measurements were only retained at times where the MARPLE output shows the average radial extent of the channel as defined by the location of the first radial inflection point of the plasma density (i.e., $\partial^{2} n_{e} / \partial r^{2}$ $=0)$ to be greater than the probe input spot size and the density to be locally minimized in the transverse plane $\left(\partial^{2} n_{e} /\left.\partial r^{2}\right|_{r=0}>0\right)$ at all points on the channel axis. Overall agreement between experimental measurements of plasma density and MARPLE simulations is excellent.

Due to mismatched guiding and self-channeling of the heater beam, $n_{e 0}$ and $r_{m}$ are not longitudinally uniform in general. This is clear in the MARPLE output shown in Fig. 4, which is for select time points of the case in Fig. 3. MARPLE simulations show that selfchanneling and mismatching of the heater beam produce a channel with a complex structure problematic for guiding at early times. Figure 4 shows that during the rise of the heater pulse at $t=-3.0 \mathrm{~ns}$, the transverse extent of the channel is small, and in some longitudinal positions, a local density maximum exists on axis. This can be seen in the transverse density profiles plotted in Fig. 4(a). The longitudinal variation of $r_{m}$ is plotted in Fig. 4(e), with values suppressed at locations with channel extents less than the probe beam waist size or with density maxima on-axis. At $t=-3.0 \mathrm{~ns}$, roughly $25 \%$ of the length of the channel meets these criteria; hence, the spot size oscillation diagnostic for $\bar{r}_{m}$ cannot be applied. Near the peak of the heater pulse at $t=1.6 \mathrm{~ns}$, a density well has formed at all points on the channel axis, but the channel remains relatively small in transverse extent and highly nonuniform in the longitudinal direction. However, several ns 

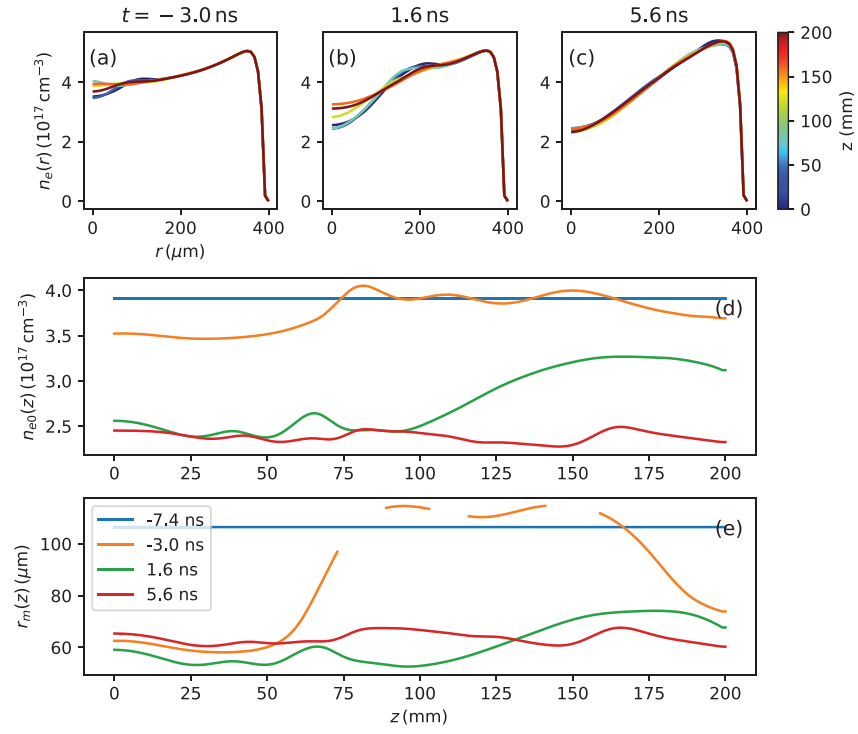

FIG. 4. MARPLE output for the case of Fig. 3. (a), (b), and (c) Radial plasma density profiles at 6 longitudinal positions $z$, for three delays from the peak of heating power: $t=-3.0 \mathrm{~ns}, t=1.6 \mathrm{~ns}$, and $t=5.6 \mathrm{~ns}$. (d) and (e) Channel parameters vs longitudinal position for $t=-7.4,-3.0,1.6,5.6 \mathrm{~ns}$, with $t=-7.4 \mathrm{~ns}$ corresponding to the unmodified discharge plasma density. $r_{m}$ values are omitted at locations where the channel extent is less than the probe beam spot size, or a density maximum exists on axis as described in Sec. IVA.

later at $t=5.6 \mathrm{~ns}$, the channel has expanded radially and become more uniform, with a longitudinal variation of $n_{e}$ and $r_{m}<10 \%$.

\section{B. Density measurement uncertainty due to channel curvature}

As stated previously in Sec. III, the asymmetry of the heater beam introduces curvature in the heated plasma channel. Evidence of this can be seen in Fig. 3(a), which shows considerable deflection of the probe beam as the channel evolves. Moreover, this deflection is very repeatable between shots, with jitter in the probe beam centroid $<10 \mu \mathrm{m}$. Overlap of the probe and heater was accurate to within
$20 \mu \mathrm{m}$ and $100 \mu \mathrm{rad}$ at the capillary entrance, which, for the measured values of $\bar{r}_{m}$, should yield a maximum deflection $<25 \mu \mathrm{m}$ and a maximum density error $<5 \times 10^{15} \mathrm{~cm}^{-3}$ from probe centroid oscillation. ${ }^{24}$ Instead, the maximum deflection observed was $175 \mu \mathrm{m}$. This is attributed to "steering" of the heater beam within the discharge channel due to the combined effects of the beam asymmetry visible in Fig. 2 and self-channeling, which creates a slightly curved plasma channel.

Consistent with the evolution of the guided probe beam during the rise of heater pulse shown in Fig. 3(a), the effect of self-channeling on the heater beam itself can be seen directly in Fig. 5, which shows the time-integrated downstream nearfield of the heater beam after propagation through a $9 \mathrm{~cm}$-long, $800 \mu$ m-diameter capillary operated at a similar initial plasma density to the case plotted in Fig. 3 as a function of laser arrival time $t_{d}$ relative to the discharge peak. The capillary discharge channel axis was aligned to the heater beam such that the paths of the guided and vacuum beam centroids overlapped within $20 \mu \mathrm{m}$ and $200 \mu \mathrm{rad}$ using the methods of Ref. 24, with a pulse energy of $30 \mathrm{~mJ}$ at delay $t_{d}=56.8 \mathrm{~ns}$ from the discharge peak.

Figure 5 shows that the heater propagation is strongly dependent on pulse energy and arrival time in the discharge pulse. At $30 \mathrm{~mJ}$, the nearfield is nearly unchanged as discharge timing is varied. This is consistent with a capillary discharge in the quasi-static regime, a condition reached near the peak of current where the plasma density profile ceases evolving and the electron temperature becomes a function of current alone, varying as $T_{e} \propto I^{1 / 2}$. However, at $420 \mathrm{~mJ}$, reductions of $\sim 3 \mathrm{mrad}$ in the divergence angle and deflections of $\sim 3 \mathrm{mrad}$ are observed when the heater pulse arrives later in the discharge. Similar to the deflection of the probe beam shown in Fig. 3(a), the changes in heater beam divergence and propagation direction are stable between shots, with jitter $<1 \mathrm{mrad}$. Increased delay relative to the discharge current peak on the falling edge of the current pulse corresponds to lower plasma temperature because of the $T_{e} \propto I^{1 / 2}$ scaling in the quasi-static regime.

That the influence of pulse energy on the propagation of the heater beam is the greatest at low plasma temperatures, where inversebremsstrahlung heating is stronger according to Eq. (5), is consistent with guiding of the heater beam within a channel created by plasma motion driven by the heater beam itself. Furthermore, deflection of the heater beam from the vacuum beam path and capillary axis at the later

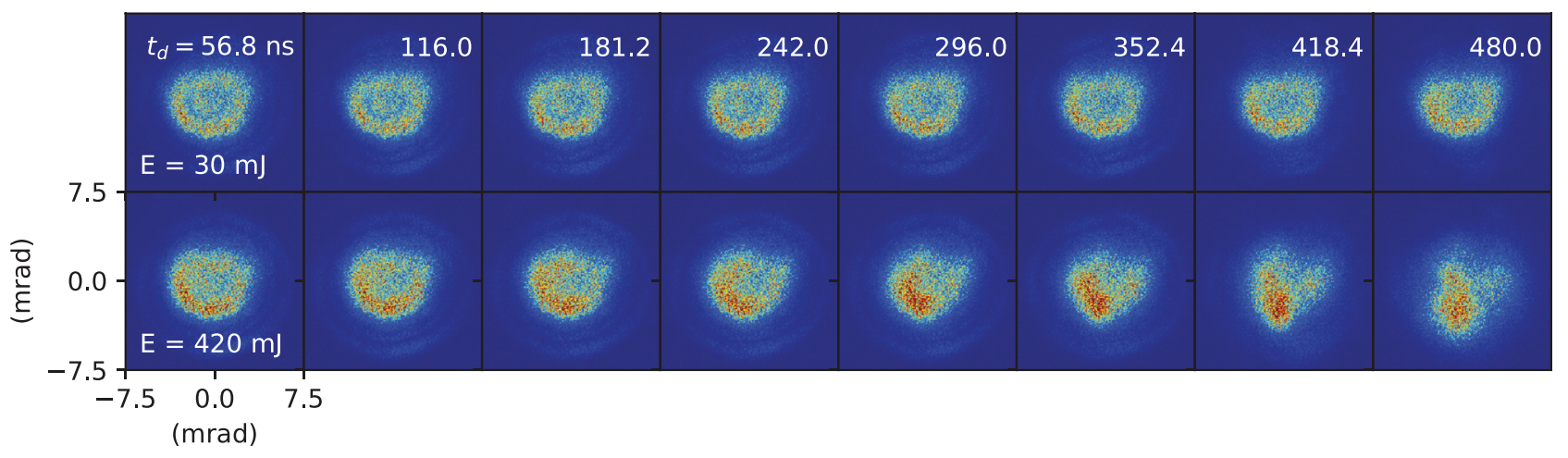

FIG. 5. Images of the heater beam downstream nearfield (imaging infinity) after interacting with a $9 \mathrm{~cm}$-long, $800 \mu$ m-diameter capillary filled with 17.2 Torr $\mathrm{H}_{2}$ vs delay $t_{d}$ of the heater pulse relative to the peak of discharge current. Images are shown for 30 and $420 \mathrm{~mJ}$ pulse energies. Both the shot-to-shot variation of the propagation direction and divergence angle are $<1$ mrad. 
times at $420 \mathrm{~mJ}$ in Fig. 5 implies that this channel is curved under these conditions.

This "self-steering" of the beam is attributed to asymmetric plasma heating, which is in turn due to the asymmetry in the heater beam shown in Fig. 2. This effect will complicate the alignment of an electron beam to the axis of an accelerator system. Thus, it is beneficial to suppress self-steering to the greatest extent possible, which requires maximizing symmetry of the heater beam. The experiments of Refs. 9 and 10 used a different laser system for heating, which produced a more symmetric beam, and self-steering was not observed.

Diagnosing the path of the curved channels formed by selfsteering is quite difficult, as discussed in Sec. III. However, assuming that guided probe beam oscillates about the center of the channel as it evolves, the maximum deflection of the probe beam in the exit plane can be used to roughly estimate a characteristic displacement of the channel from the capillary axis. For the case of Fig. 3, taking the maximum probe deflection of $\Delta x_{f}=175 \mu \mathrm{m}$ as the peak-to-peak oscillation amplitude about the channel center yields the estimate $\Delta x_{f} / 2 \approx 90 \mu \mathrm{m}$ for the displacement of the channel center from the capillary axis at the exit plane. This value is merely representative, however. The position of the channel center varies with the longitudinal position along the capillary and in fact coincides with the capillary axis at the entrance plane to within the alignment precision of $20 \mu \mathrm{m}$ previously quoted.

The main difficulty introduced in this experiment by self-steering of the heater beam is a systematic error in the density measurement, caused by the increased path length traversed by the probe pulse in a curved channel. The wide-cap error bars of Fig. 3(b) denote the uncertainty in plasma density due to channel curvature and finite matched spot size effects. Uncertainty from jitter in the relative group delay of the probe and reference pulses is denoted by the narrow-cap error bars. The wide-cap error bars were calculated using measurements of $\bar{r}_{m}$ in combination with the observed maximum deflection of the probe beam, $\Delta x_{f}=175 \mu \mathrm{m}$ (see Appendix B). Because these propagation effects scale roughly as $r_{m}^{-2}$, they are most pronounced close to the peak of the heater pulse, where $\bar{r}_{m}$ is the smallest.

The peak in $\bar{n}_{e 0}$ visible in the experimental data plotted in Fig. 3(b) at $t \sim-4$ ns is a diagnostic artifact rather than a physical increase in the plasma density. Measurements on the BELLA main beamline ${ }^{9,10}$ using a different diagnostic technique, common-path two-color spectral interferometry, ${ }^{30,31}$ agreed well with the simulations and measurements reported in this paper but did not observe this feature. Moreover, ionization by the heater laser cannot account for the observed effect, as MARPLE shows the fraction of neutral hydrogen on axis to be $\$ 2 \%$, ${ }^{10}$ leaving insufficient neutral hydrogen to produce the observed $\sim 25 \%$ increase in apparent electron density.

In Fig. 3(b), the simulated density lies within the systematic uncertainty from propagation effects, with the exception of the points at $t=-6 \mathrm{~ns}$ and $-4 \mathrm{~ns}$. The discrepancy at these times may arise from the assumption of an ideal, longitudinally uniform parabolic channel in the model of the decreased group velocity and increased path length due to finite matched spot size and channel curvature (see Appendix B). As previously discussed, the MARPLE simulations show that early in the heater pulse, the channel is both longitudinally nonuniform and has a complex, non-parabolic transverse profile. In addition to having these properties, the physical channel is non-axisymmetric. The group velocity of the probe pulse in such a structure will be less than in the idealized parabolic density profile used in the model, in which case the apparent density contribution from propagation effects will be underestimated.

Nevertheless, the group delay plasma density measurements agree well with simulation in the interval most useful for laser-plasma acceleration $0<t<15 \mathrm{~ns}$, when the channel is fully formed and onaxis density and matched spot size are low. Figure 4 shows that plasma expansion produces a transverse density profile with a wide, on-axis minimum by $t \approx 2 \mathrm{~ns}$, which further evolves into a longitudinally uniform, nearly parabolic shape on-axis by $t \approx 6 \mathrm{~ns}$. Additionally, the effects of matched spot size and channel curvature are greatly reduced at later times due to increasing $\bar{r}_{m}$ after $t \approx 0$ ns and the $r_{m}^{-2}$ scaling of these effects. Therefore, the diagnostic artifacts and nonidealities of the plasma profile previously discussed pose little difficulty for measurements in the interval relevant to accelerator operation.

The measurements and simulation output of Figs. 3 and 4 show that, for a given capillary radius, laser heating of a capillary discharge can create a channel with lower matched spot size for a given density than the discharge alone. Equivalently, channels can be created with matched spot size and density that would otherwise require a significantly smaller capillary radius without laser heating: $60 \%$ smaller in the case of Figs. 3 and 4. The properties of the channel, including $\bar{n}_{e 0}, \bar{r}_{m}$, and the longitudinal variation of the plasma profile, evolve on a nanosecond timescale due to plasma motion. This requires precise timing of the driver pulse to achieve optimal channel properties, with a minimum in on-axis density occurring $\sim 5 \mathrm{~ns}$ after the peak of the heater pulse. Furthermore, MARPLE simulations showing $\sim 10 \%$ longitudinal variation in $\bar{r}_{m}$ and $\bar{n}_{e 0} \sim 6 \mathrm{~ns}$ after the peak demonstrate that, despite mismatch of the heater spot size to the channel and the complex coupling between plasma motion and heater beam propagation, channels with a high degree of longitudinal uniformity can be created.

\section{TUNING CHANNEL PROPERTIES VIA PLASMA AND LASER PARAMETERS}

The primary difficulty addressed by laser-heated capillary discharges is the lack of independent control of $r_{m}$ and $n_{e 0}$ in a conventional capillary discharge. For a capillary discharge, the scaling $r_{m} \propto r_{c}^{1 / 2} n_{e 0}^{-1 / 4}$ restricts a capillary of the fixed radius to a onedimensional curve in $\left(n_{e 0}, r_{m}\right)$ space. Independent control of $n_{e 0}$ and $r_{m}$ enables matched guiding of petawatt-scale laser pulses at low plasma densities required for $\sim 10 \mathrm{GeV}$ energy gain without reducing the capillary diameter to a size at which the structure is subject to laser damage. This additional freedom is also useful for optimization of a channel-guided laser-plasma accelerator, where matched spot size, plasma density, laser intensity, and plasma wave phase velocity are all coupled to one another.

It was demonstrated in Ref. 9 that a laser-heated capillary discharge can be tuned over a two-dimensional region of $\left(\bar{n}_{e 0}, \bar{r}_{m}\right)$ by varying the initial discharge plasma density and temperature. In the experiments of Ref. 9, the driver arrived at the peak of the heater pulse and channel parameters were reported for that delay relative to the heater pulse. Here, the full time evolution of the plasma upon heating is presented for the parameter space discussed in Ref. 9, as well as additional measurements demonstrating the effect of the total heater pulse energy. In agreement with the data of Fig. 3, the data presented here show that a minimum in the on-axis density consistently occurs 
4-7 ns after the peak of the heater pulse, indicating that for a wide range of parameters, channel properties are optimal for high energy gain at that time. Tunability of channel properties over a wide range via heater laser energy, driver timing, and initial plasma parameters is shown, and strategies for channel optimization are identified.

\section{A. Initial plasma density}

Experiments and MARPLE simulations show that the reduction of $\bar{n}_{e 0}$ and $\bar{r}_{m}$ increases with initial plasma density. Figure 6 shows $\bar{n}_{e 0}$ and $\bar{r}_{m}$ plotted for a heater laser arrival time $t_{d}=320 \mathrm{~ns}$ after the discharge peak for four initial plasma densities $\bar{n}_{e 0, i}$. Reduction of $\bar{n}_{e 0}$ and $\bar{r}_{m}$ from their initial values is found to increase with $\bar{n}_{e 0, i}$. This relationship is to be expected from the $n_{e}$ scaling of the heating rate [Eq. (5)]. Low densities and matched spot sizes cannot be achieved by simply lowering the initial plasma density because the modification of $\bar{r}_{m}$ decreases rapidly with density. Instead, there exists an optimal initial density for guiding and acceleration that produces the required reduction in both $\bar{r}_{m}$ and $\bar{n}_{e 0}$.
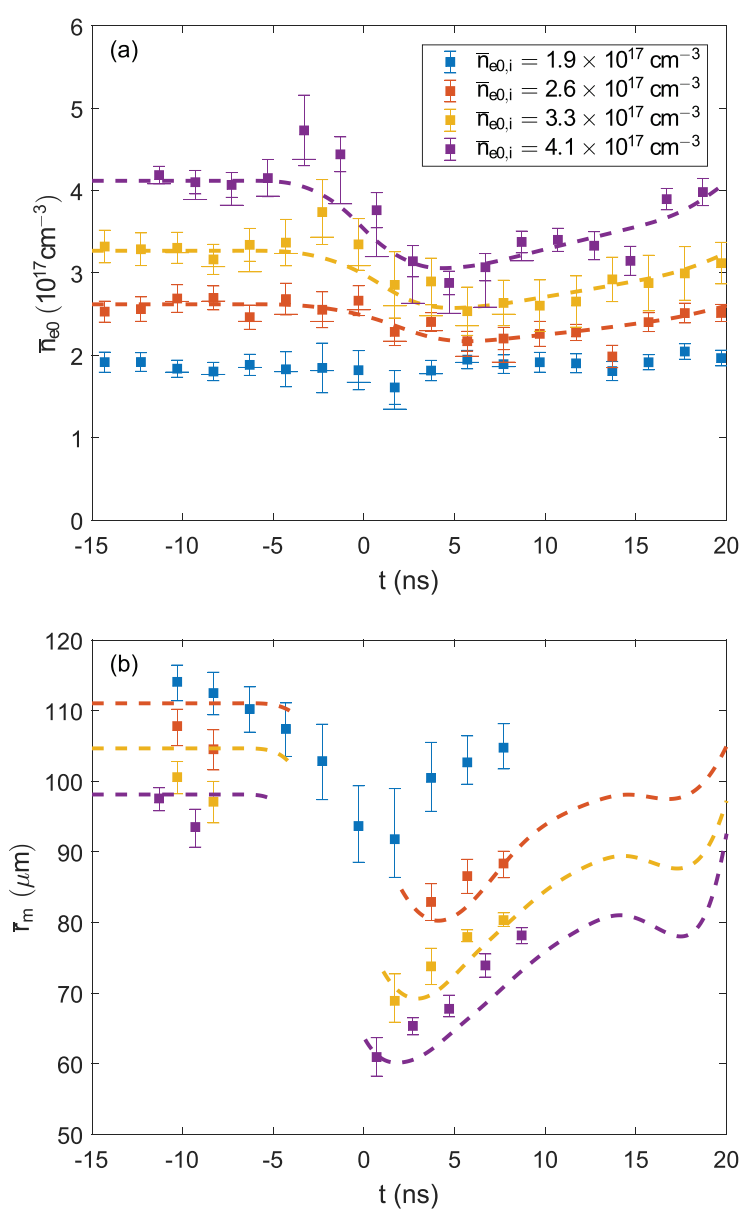

FIG. 6. (a) $\bar{n}_{e 0}$ for four initial densities, with the arrival time of the heater pulse relative to peak discharge current $t_{d}=320 \mathrm{~ns}$. Error bars are as in Fig. 3. Values from MARPLE are dotted lines. (b) Measured $\bar{r}_{m}$ and MARPLE calculated values for the cases of (a)

\section{B. Initial plasma temperature}

Initial plasma temperature $T_{e, i}$ has a strong effect on plasma profile modification. Figure 7 shows $\bar{n}_{e 0}$ and $\bar{r}_{m}$ for three different arrival times of the heater pulse relative to the discharge peak, which correspond to different initial plasma temperatures $T_{e, i}$. As previously discussed, plasma temperature decreases with delay from the peak of discharge current because of the $T_{e} \propto I^{1 / 2}$ scaling in the quasi-static regime. ${ }^{7}$ Lower $T_{e, i}$ at later times yields larger reductions in both $\bar{n}_{e 0}$ and $\bar{r}_{m}$ due to the $T_{e}^{-3 / 2}$ scaling of the heating rate [Eq. (5)].

Initial plasma temperature is the most readily exploited parameter for optimization of the heated channel because it can be easily tuned while minimally altering other discharge channel properties. Once the quasi-static regime is reached near the peak of the current pulse, the shape of the plasma profile does not evolve significantly. Centroid oscillation measurements show the matched spot size $r_{m}$ of the capillary discharge channel (without heating) varied $<2 \mu \mathrm{m}$ between the three delays shown in Fig. 7, which cover an interval of 420 ns.
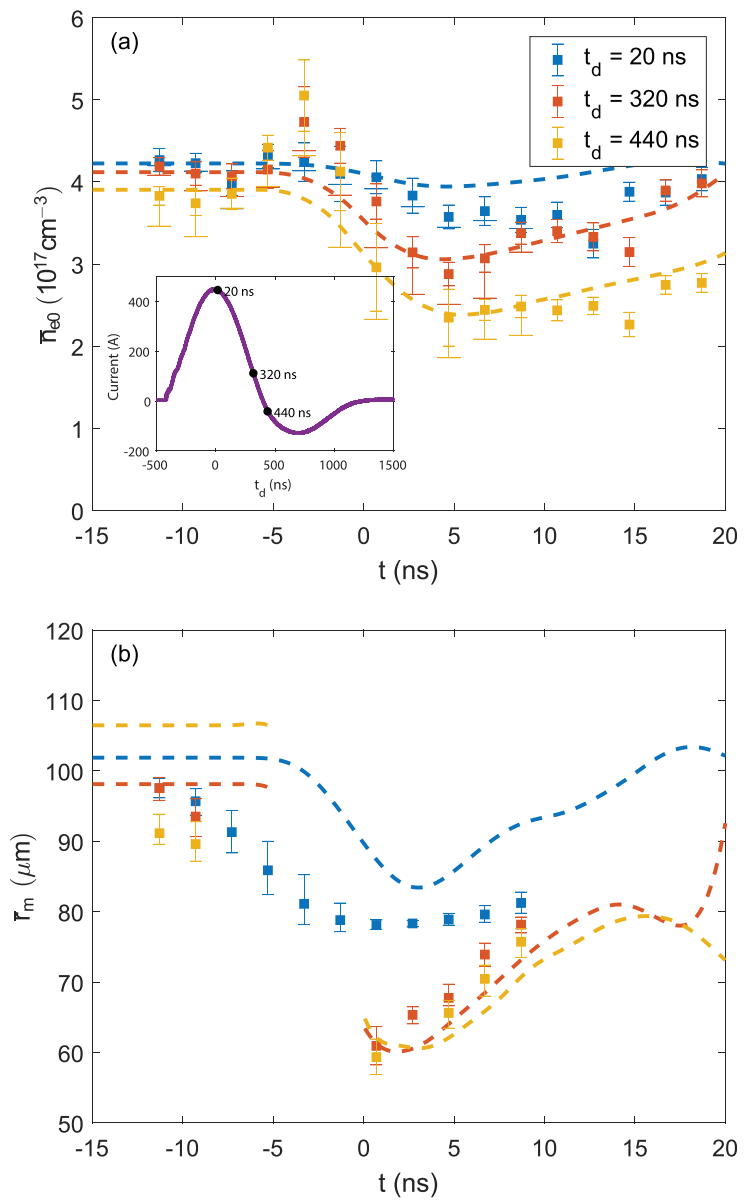

FIG. 7. (a) $\bar{n}_{e 0}$ for three arrival times of the heater pulse relative to the discharge current pulse. Heater pulse arrival times $t_{d}$ are marked on the discharge current trace in the inset. Later times and lower currents correspond to lower initial plasma temperature. Error bars are as in Fig. 3. Values from MARPLE are dotted lines. (b) Measured $\bar{r}_{m}$ and MARPLE calculated values for the cases of (a). 
However, the requirement of near-total ionization on-axis for acceleration imposes a minimum allowable initial temperature and therefore maximum allowable laser delay from the peak of discharge current. If the neutral fraction becomes too great, ionization defocusing of the driver can overwhelm the effect of the channel. Laser heating can ionize a partially recombined plasma at late times in the discharge current pulse, and therefore, the plasma is not necessarily required to be initially fully ionized. The maximum allowable delay from the peak of current is, therefore, dependent on the parameters of the capillary discharge and the heater laser. For these experiments, ionization blueshifting measurements and MARPLE simulations indicate that up to $420 \mathrm{~ns}$ after the peak of discharge current, the plasma on the channel axis is fully ionized $\sim 5 \mathrm{~ns}$ after the peak of a $300 \mathrm{~mJ}$ heater pulse. ${ }^{10}$

\section{Heater pulse energy}

It might be expected that increasing the energy of the heater pulse would be a straightforward means of decreasing on-axis density and matched spot size. However, the plasma response was observed to saturate with increasing pulse energy. Figure 8 shows $\bar{n}_{e 0}$ and $\bar{r}_{m}$ measurements at four heater pulse energies for $\bar{n}_{e 0, i}=3.8 \times 10^{17} \mathrm{~cm}^{-3}$ and laser timing $t_{d}=440 \mathrm{~ns}$ after the peak of discharge current. Measurable modification of the plasma occurs with as few as $30 \mathrm{~mJ}$ of laser energy, $10 \%$ of the pulse energy that was used in the LPA experiments of Refs. 9 and 10. The plasma modification observed at $30 \mathrm{~mJ}$ is strong evidence for the influence of self-channeling on the propagation of the heater pulse at higher energies: based on the effect observed at $30 \mathrm{~mJ}$, the rising edge of a joule-level pulse contains sufficient energy to significantly modify the plasma profile before the majority of the pulse energy has been deposited.

The effect of laser-heating begins to saturate above energies of $100 \mathrm{~mJ}$. Measurements of $\bar{n}_{e 0}$ and $\bar{r}_{m}$ cannot distinguish between channel properties for the cases of 200 and $300 \mathrm{~mJ}$ of laser energy, although the MARPLE simulations show a slightly lower density for $300 \mathrm{~mJ}$. This saturation with heater pulse energy is a consequence of the $T_{e}^{-3 / 2}$ scaling of the heating rate [Eq. (5)]. As the plasma absorbs energy from the heater pulse and $T_{e}$ increases, it becomes more transparent.

Saturation of the plasma response with respect to pulse energy is predicted by a simplified model of heating as a function of the fluence $\Phi \equiv \int_{-\infty}^{t} I \mathrm{~d} t^{\prime}$ derived from Eq. (3). Taking the factors $\Lambda$ and $n_{e}$ constant in Eq. (3) and ignoring heat conduction, solving the resulting differential equation yields

$$
T_{e}=\left(K n_{e} \Phi \Lambda / \omega^{2}+T_{e, i}^{5 / 2}\right)^{2 / 5},
$$

with $T_{e, i}$ the initial plasma temperature and

$$
K=\frac{2^{7 / 2} \pi^{3 / 2}}{3} \frac{Z q_{e}^{6}}{k_{b}^{5 / 2} m_{e}^{3 / 2} c}
$$

a proportionality constant composed of dimensionless factors and physical constants from Eqs. (3) and (4). Note that as a consequence of neglecting plasma motion and heat conduction, the expression for $T_{e}$ is independent of the temporal structure of the laser intensity $I$, depending only on the fluence $\Phi$. This follows from the linearity of the heating rate in the intensity. The saturation behavior is evident from the fact $\mathrm{d}^{2} T_{e} / \mathrm{d} \Phi^{2}<0$. In reality, this simplified model overestimates
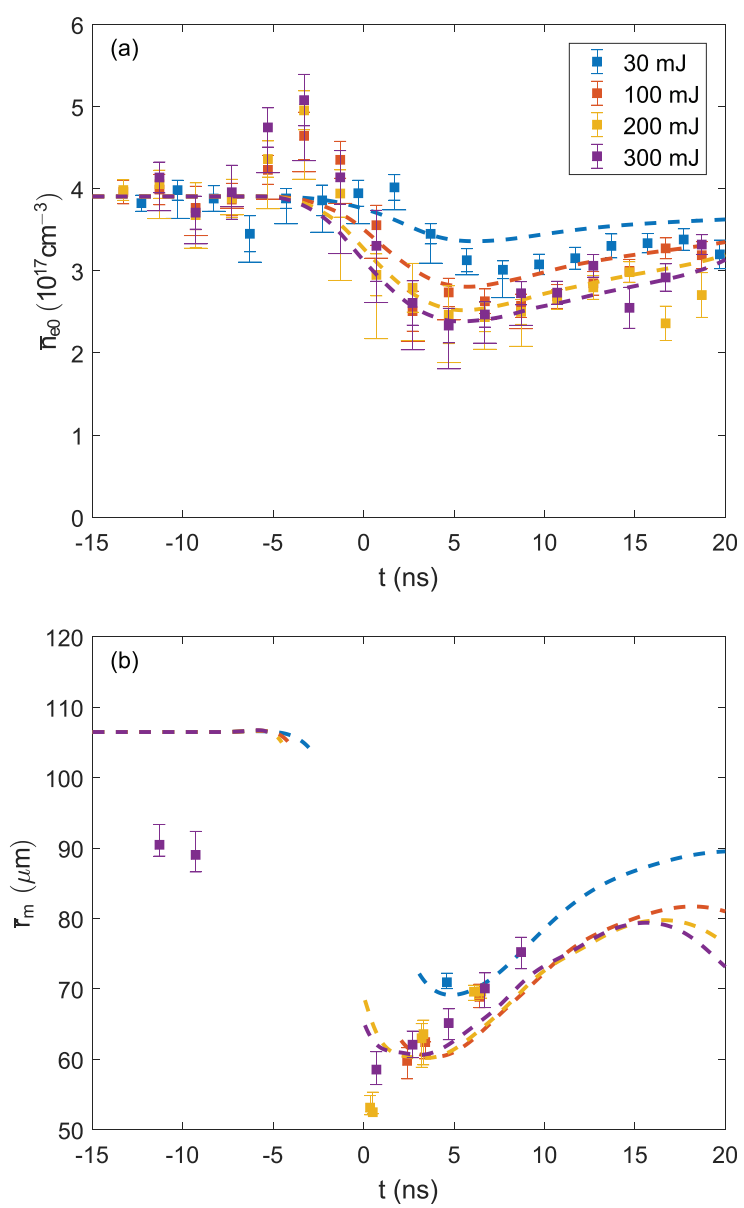

FIG. 8. (a) $\bar{n}_{e 0}$ for four heater pulse energies, with identical initial plasma temperature and density. The initial plasma density is $3.8 \times 10^{17} \mathrm{~cm}^{-3}$, and the arrival time of the heater relative to peak discharge current is $t_{d}=440 \mathrm{~ns}$. Error bars are as in Fig. 3. Values from MARPLE are dotted lines. (b) Measured $\bar{r}_{m}$ and MARPLE calculated values for the cases of (a).

heating for the experiments reported in this paper, as plasma motion will reduce $n_{e}$ during heating, and heat conduction will allow energy to migrate out of the heated region of the plasma. Therefore, plasma temperature will saturate more rapidly than the $\sim \Phi^{2 / 5}$ scaling of Eq. (6).

It is also evident from Eq. (6) and its derivation from Eq. (3) that sensitivity of the plasma response to the temporal structure of the heater pulse arises through plasma motion and heat conduction, provided that the laser intensity remains low enough that the inversebremsstrahlung heating rate remains linear in the intensity. Although not directly applicable to the experiments in this paper, this may be an important consideration in situations where the heater pulse length is shorter than the characteristic timescales of these processes.

However, the heating rate [Eq. (3)] will become nonlinear in the intensity if the electron ponderomotive energy becomes comparable to the plasma temperature because the collision frequency $\nu_{e, I B}$ will begin to decrease with laser intensity. ${ }^{15,16}$ In this regime, Eq. (6) will no longer hold and $T_{e}$ will become dependent on the heater pulse shape. For the laser and plasma parameters considered in this paper, this occurs 
at a laser intensity and a pulse energy of order $10^{14} \mathrm{~W} / \mathrm{cm}^{2}$ and $100 \mathrm{~J}$, respectively. Moreover, Eq. (6) does not hold for heater pulse lengths shorter than the electron-ion energy exchange timescale regardless of intensity, as the approximation $T_{e} \approx T_{i}$ from which Eq. (3) is derived is no longer valid. Therefore, per the discussion in Sec. II, Eq. (6) is not valid for heater pulse lengths of order 100 ps and shorter for the plasmas considered here.

Measurements and simulations show that for laser-heated capillary discharges, channel properties are sensitive to initial plasma density and temperature, delay from the heater pulse, and heater pulse energy. This enables independent tuning of $\bar{n}_{e 0}$ and $\bar{r}_{m}$ and enables optimization of guiding with a fixed capillary radius. For example, MARPLE simulations show matched spot sizes of 75 and $65 \mu \mathrm{m}$ at a density of $2.4 \times 10^{17} \mathrm{~cm}^{-3}: 75 \mu \mathrm{m}$ at a discharge timing and an initial density of $t_{d}=320 \mathrm{~ns}$ and $\bar{n}_{e 0, i}=3.3 \times 10^{17} \mathrm{~cm}^{-3}$ (Fig. 6) and 65 $\mu \mathrm{m}$ at $t_{d}=440 \mathrm{~ns}$ and $\bar{n}_{e 0, i}=3.8 \times 10^{17} \mathrm{~cm}^{-3}$ (Fig. 6). To vary matched spot size over the same interval with a capillary discharge, the scaling $r_{m} \propto r_{c}^{1 / 2} n_{e}^{-1 / 4}$ would require a $50 \%$ increase in density for a fixed capillary radius or a $25 \%$ decrease in the capillary diameter for fixed density.

Density can be tuned while the matched spot is kept fixed as well. The MARPLE simulations of Fig. 8 show that the density can be tuned between $3.4 \times 10^{17}$ and $2.5 \times 10^{17} \mathrm{~cm}^{-3}$ at a constant matched spot size of $69 \mu \mathrm{m}$ by varying laser energy between 30 and $300 \mathrm{~mJ}$ and delay from the heater pulse peak between 5 and 8 ns. Simulations show that single-stage acceleration to energies approaching $10 \mathrm{GeV}$ is possible within this space of accessible spot sizes and densities, ${ }^{28}$ and tunability of these parameters independent of the capillary radius adds useful flexibility over a conventional capillary discharge, especially when the capillary radius is restricted by other factors such as laser damage.

\section{CONCLUSION}

Laser-heated capillary discharge waveguides have been characterized as novel, tunable guiding structures for LPAs. The independent control of matched spot size and plasma density afforded by these structures has enabled guiding of petawatt-scale pulses focused to a beam waist of $\sim 60 \mu \mathrm{m}$ at low plasma densities required for the production of multi-giga-electron volt electron beams within capillaries of a large enough diameter to avoid laser damage. ${ }^{9,10}$ This independent tunability of matched spot and density is also useful for the optimization of channel-guided LPAs, where one of these parameters may be constrained by such considerations as required beam energy or control of bunch injection. In this paper, important trends in the properties of laser-heated capillary discharge waveguides have been identified. Guiding and acceleration can be tuned via initial plasma density and temperature, heater laser pulse energy, and arrival time of the driver relative to the heater pulse. Trends observed in these experiments are consistent with theoretical models of low power inversebremsstrahlung, and specific experimental measurements are well reproduced by the MARPLE MHD code. Importantly, the measurements of the time evolution of the plasma channel reported here indicate a path toward improved accelerator performance over previous experiments $^{9,10}$ through optimizing the arrival time of the driver relative to the heater pulse.

Future experiments and simulations with MARPLE will investigate additional strategies for optimizing channel performance through tuning of the heater beam focal spot size, pulse length, and wavelength, which are known to be important parameters for laser-heated capillary discharge waveguides.

\section{ACKNOWLEDGMENTS}

This work was supported by the Director, Office of Science, Office of High Energy Physics, of the U.S. Department of Energy under Contract Nos. DE-AC02-05CH11231 and DEFG02-12ER41798, the National Science Foundation under Grant Nos. PHY-1415596 and PHY-1632796, the project High Field Initiative (No. CZ.02.1.01/0.0/0.0/15_003/0000449) from the European Regional Development Fund, and RFBR Project No. 20-31-70015. This work used resources of the National Energy Research and Scientific Computing Center (NERSC), a U.S. Department of Energy Office of Science User Facility. The authors gratefully acknowledge technical support from Zac Eisentraut, Dave Evans, Mark Kirkpatrick, Art Magana, Greg Mannino, Joe Riley, Tyler Sipla, Will Waldron, Don Syversrud, and Nathan Ybarrolaza.

\section{DATA AVAILABILITY}

The data that support the findings of this study are available from the corresponding author upon reasonable request.

\section{APPENDIX A: MATCHED SPOT SIZE DIAGNOSTIC}

A Gaussian laser beam propagating in an longitudinally uniform parabolic channel will undergo oscillations in spot size according to ${ }^{23,24}$

$$
r_{s}^{2}=\frac{r_{i}^{2}}{2}\left[1+\frac{r_{m}^{4}}{r_{i}^{4}}+\left(1-\frac{r_{m}^{4}}{r_{i}^{4}}\right) \cos \left(2 k_{\beta c} z\right)\right],
$$

with $k_{\beta c}=2 / k r_{m}^{2}$. This oscillation can be used as a diagnostic for $\bar{r}_{m}$, by imaging the beam at the capillary exit as $\bar{r}_{m}$ is varied. The peak intensity of the probe beam at the capillary exit will oscillate as $\bar{r}_{m}$ is varied, and so, an oscillating peak intensity "trace" is obtained. The extrema of the peak intensity trace occur at values of $\bar{r}_{m}$ determined by the input beam parameters. In the experiments discussed here, $\bar{r}_{m}$ was varied by adjusting the fill pressure of the capillary, while the probe delay relative to the heater pulse was held constant.

To identify the $\bar{r}_{m}$ values for extrema in the peak intensity trace, the peak intensity must include a pressure with a known value of $\bar{r}_{m}$. This was accomplished by beginning each peak intensity trace at very low fill pressure, where the heater has a negligible effect on the plasma profile and the matched spot size is unchanged from that of the discharge without heating. Therefore, measurements of $\bar{r}_{m}$ for the discharge alone using probe centroid oscillations were used to determine $\bar{r}_{m}$ at the low pressure end of the peak intensity trace and the fact that $\bar{r}_{m}$ decreases with pressure was used to identify the $\bar{r}_{m}$ values of the extrema in the trace as pressure was increased. Values of $\bar{r}_{m}$ at points between extrema in the peak intensity traces were interpolated. Uncertainties in the measurements of $\bar{r}_{m}$ were calculated from the uncertainties in fill pressure and probe delay associated with each of the extrema in the peak intensity traces.

Because the probe beam used in these experiments was nonGaussian, a more detailed model for the spot size oscillation than 
Eq. (A1) was required to assign $\bar{r}_{m}$ values to the extrema in the peak intensity traces. To facilitate this, a model of the probe beam field was constructed using a wavefront measurement. This model of the probe beam was used as input to the INF\&RNO code, ${ }^{26}$ where it was propagated through a set of $20 \mathrm{~cm}$-long parabolic channels with $r_{m}$ ranging from 40 to $120 \mu \mathrm{m}$. The INF\&RNO simulations were used to calculate the peak irradiance at the channel exit as a function of $\bar{r}_{m}$, and the extrema of this function were mapped to the experimental peak intensity traces.

\section{APPENDIX B: DENSITY MEASUREMENT UNCERTAINTY DUE TO FINITE MATCHED SPOT SIZE AND CHANNEL CURVATURE}

Finite matched spot size and misalignment due to channel curvature increase the apparent on-axis density obtained from the group velocity measurements reported in this paper. These effects are treated as a systematic error in the density measurements, denoted by the wide-cap error bars of Figs. 3 and 6-8. In this Appendix, the model used to estimate this error is described in detail.

In the experiments here, the group velocity of the probe pulse centroid was used to measure the on-axis plasma density $\bar{n}_{e 0}$. In practice, the average group velocity is obtained by measuring the additional group delay induced by propagation through the channel as compared to vacuum,

$$
\Delta T \equiv T-T_{v a c}=\frac{1}{c} \int_{0}^{L}\left(\frac{1}{\beta_{g}}-1\right) \mathrm{d} z,
$$

with $L$ the path length traversed in the channel, which is typically the capillary length. The group velocity $\beta_{g}$ of the centroid of a laser pulse propagating in an longitudinally uniform parabolic channel is given by the following formula: ${ }^{27}$

$$
\beta_{g}=\frac{v_{g}}{c} \simeq 1-\beta_{g, n_{e}}-\beta_{g, g e o},
$$

with the component $\beta_{g, n_{e}}=k_{p}^{2} / 2 k^{2}$ from the on-axis plasma density $n_{e 0}$ and the component

$$
\begin{aligned}
\beta_{g, g e o}(z)= & \frac{2}{k^{2} r_{m}^{2}}\left\{\frac{r_{0}^{2} r_{m}^{2} \sec ^{2}\left(k_{\beta c} z\right)}{r_{0}^{4}+r_{m}^{4} \tan ^{2}\left(k_{\beta c} z\right)}\right. \\
& \left.+\frac{2 r_{0}^{2} r_{m}^{2}\left(r_{m}^{4}-r_{0}^{4}\right)\left(k_{\beta c} z\right) \tan \left(k_{\beta c} z\right) \sec ^{2}\left(k_{\beta c} z\right)}{\left[r_{0}^{4}+r_{m}^{4} \tan ^{2}\left(k_{\beta c} z\right)\right]^{2}}\right\}
\end{aligned}
$$

from the finite matched spot size of the channel. The total contribution of $\beta_{g, n_{e 0}}$ and $\beta_{g, g e o}$ is typically $\lesssim 10^{-4}$, and so, Eq. (B1) can be simplified to the following expression, substituting in Eq. (B2):

$$
\Delta T \simeq \frac{1}{c} \int_{0}^{L}\left(\beta_{g, n_{e 0}}+\beta_{g, g e o}\right) \mathrm{d} z,
$$

yielding

$$
\Delta T \simeq \frac{L}{c}\left(\bar{\beta}_{g, n_{e 0}}+\bar{\beta}_{g, g e o}\right) .
$$

The quantity of interest for $n_{e 0}$ is $\bar{\beta}_{g, n_{e 0}}$, and so, $\bar{\beta}_{g, g e o}$ must be determined and its contribution to the group velocity is subtracted from Eq. (B4). This requires an accurate measurement of $r_{m}$ and can be readily accomplished for capillary discharges using the methods previously discussed from Ref. 24. However, the channels formed by laser heating can be significantly longitudinally nonuniform, and so, Eq. (B3) is approximate at best. Because of the lack of diagnostics for the longitudinal variation of $r_{m}$, the effects of finite channel matched spot size were treated as a systematic error in the density measurement, estimated with Eq. (B3) using $\bar{r}_{m}$ values from spot size oscillations.

As discussed in Sec. IV, asymmetry in the heater beam leads to the creation of curved plasma channels misaligned to the probe beam and the capillary axis. This misalignment increases the path length traveled by the probe beam and thus the apparent plasma density. The channel's curvature itself contributes little to the path length of the probe beam through the plasma but ultimately leads to misalignment of the probe from the channel center, resulting in centroid oscillation of the probe beam. This oscillation contributes significant path length and increases the apparent plasma density by roughly the same amount as finite spot size effects.

The path length contributed by this misalignment was estimated by modeling the channel produced by laser heating as a straight parabolic waveguide, with the probe beam injected at the channel center in the entrance plane but at a nonzero angle $\theta_{i}$ relative to the axis. This geometry does not reflect the curved shape of the channel, which is difficult to diagnose directly and nevertheless contributes a negligible path length. However, this model is a useful approximation with which the probe beam path length contributed by centroid oscillations can be estimated analytically while preserving some physical features of the probe beam's coupling into the channel, namely, the initial overlap of the probe and heater beams at the capillary entrance plane. In the model, the total path length traveled by the probe beam is then

$$
L=L_{0}+\delta L=\int_{0}^{L_{0}} \sqrt{1+\left(\frac{d x}{d z}\right)^{2}} \mathrm{~d} \mathrm{z}
$$

with $L_{0}$ the capillary length. The trajectory of the probe centroid according to Refs. 23 and 24 is

$$
x=x_{i} \sin \left(k_{\beta c} z\right),
$$

with $x_{i}=\theta_{i} / k_{\beta c}$ and $k_{\beta c}=2 / k r_{m}^{2}$. Because the additional path length from the centroid oscillation is small compared to the capillary length (at most $10 \mu \mathrm{m}$ ), $\delta L$ is well approximated by

$$
\delta L \simeq \int_{0}^{L_{0}} \frac{1}{2}\left(\frac{d x_{i}}{d z}\right)^{2} \mathrm{~d} z .
$$

Using Eq. (B6) in Eq. (B7) and integrating, we get

$$
\delta L \simeq \frac{1}{2} L_{0} k_{\beta c}^{2} x_{i}^{2}\left[\frac{1}{2}-\frac{\cos \left(2 k_{\beta c} L_{0}\right)}{4 k_{\beta c} L_{0}}\right] .
$$

The model has two free parameters, $r_{m}$ and $x_{i}$, which must be determined by external measurements. The matched spot size $r_{m}$ is set to $\bar{r}_{m}$ as measured with the diagnostic techniques described elsewhere. The oscillation amplitude $x_{i}$ is estimated as half the greatest distance between two probe centroid positions over the course of 
the plasma evolution. For the case of Fig. 3, this distance is $175 \mu \mathrm{m} / 2=87.5 \mu \mathrm{m}$.

The additional path length $\delta L$ can be incorporated into the model for channel induced delay [Eq. (B4)] by expanding around $L_{0}$ in Eq. (B1),

$$
\int_{0}^{L_{0}+\delta L} \frac{1}{\beta_{g}} \mathrm{dz} \simeq \int_{0}^{L_{0}} \frac{1}{\beta_{g}} \mathrm{dz}+\frac{\delta L}{\beta_{g}} .
$$

Taking $\beta_{g} \simeq 1$ in the second term on the RHS of Eq. (B9), substituting through Eqs. (B1) and (B4), the following equation for $\bar{\beta}_{g, n_{e 0}}$ is obtained:

$$
\bar{\beta}_{g, n_{e 0}}=\frac{c \Delta T}{L_{0}}-\bar{\beta}_{g, g e o}-\frac{\delta L}{L_{0}} .
$$

This equation includes the effects of both finite matched spot size and channel curvature in terms $-\bar{\beta}_{\text {g.geo }}$ and $-\delta L / L_{0}$, respectively. These two terms are always negative and therefore define the lower bound on the plasma density for the measured channel induced delay $\Delta T$ and are treated as a systematic error. As discussed in Sec. IV, $\bar{r}_{m}$ measurements are omitted where MARPLE simulations show the channel radius to be less than the probe input spot size. However, these omitted values were used to calculate the wide-cap error bars from the finite matched spot size and channel curvature effects.

\section{REFERENCES}

${ }^{1}$ E. Esarey, C. B. Schroeder, and W. P. Leemans, Rev. Mod. Phys. 81, 1229 (2009).

${ }^{2}$ W. P. Leemans, B. Nagler, A. J. Gonsalves, C. Tóth, K. Nakamura, C. G. R. Geddes, E. Esarey, C. B. Schroeder, and S. M. Hooker, Nat. Phys. 2, 696 (2006).

${ }^{3}$ T. P. A. Ibbotson, N. Bourgeois, T. P. Rowlands-Rees, L. S. Caballero, S. I. Bajlekov, P. A. Walker, S. Kneip, S. P. D. Mangles, S. R. Nagel, C. A. J. Palmer, N. Delerue, G. Doucas, D. Urner, O. Chekhlov, R. J. Clarke, E. Divall, K. Ertel, P. S. Foster, S. J. Hawkes, C. J. Hooker, B. Parry, P. P. Rajeev, M. J. V. Streeter, and S. M. Hooker, Phys. Rev. Spec. Top. Accel. Beams 13, 031301 (2010).

${ }^{4}$ D. J. Spence and S. M. Hooker, Phys. Rev. E 63, 015401 (2000).

${ }^{5}$ W. P. Leemans, A. J. Gonsalves, H.-S. Mao, K. Nakamura, C. Benedetti, C. B. Schroeder, C. Tóth, J. Daniels, D. E. Mittelberger, S. S. Bulanov, J.-L. Vay, C. G. R. Geddes, and E. Esarey, Phys. Rev. Lett. 113, 245002 (2014).

${ }^{6}$ C. Benedetti, F. Rossi, C. B. Schroeder, E. Esarey, and W. P. Leemans, Phys, Rev. E 92, 023109 (2015).

${ }^{7}$ N. A. Bobrova, A. A. Esaulov, J.-I. Sakai, P. V. Sasorov, D. J. Spence, A. Butler, S. M. Hooker, and S. V. Bulanov, Phys. Rev. E 65, 016407 (2001)

${ }^{8}$ N. A. Bobrova, P. V. Sasorov, C. Benedetti, S. S. Bulanov, C. G. R. Geddes, C. B. Schroeder, E. Esarey, and W. P. Leemans, Phys. Plasmas 20, 020703 (2013).
${ }^{9}$ A. J. Gonsalves, K. Nakamura, J. Daniels, C. Benedetti, C. Pieronek, T. C. H. de Raadt, S. Steinke, J. H. Bin, S. S. Bulanov, J. van Tilborg, C. G. R. Geddes, C. B. Schroeder, C. Tóth, E. Esarey, K. Swanson, L. Fan-Chiang, G. Bagdasarov, N. Bobrova, V. Gasilov, G. Korn, P. Sasorov, and W. P. Leemans, Phys. Rev. Lett. 122, 084801 (2019)

${ }^{10}$ A. J. Gonsalves, K. Nakamura, C. Benedetti, C. V. Pieronek, S. Steinke, J. H. Bin, S. S. Bulanov, J. van Tilborg, C. G. R. Geddes, C. B. Schroeder, J. Daniels, C. Tóth, L. Obst-Huebl, R. G. W. van den Berg, G. Bagdasarov, N. Bobrova, V. Gasilov, G. Korn, P. Sasorov, W. P. Leemans, and E. Esarey, Phys. Plasmas 27, 053102 (2020).

${ }^{11}$ V. Gasilov, A. Boldarev, S. Dyachenko, O. Olkhovskaya, E. Kartasheva, G. Bagdasarov, S. Boldyrev, I. Gasilova, V. Shmyrov, S. Tkachenko, J. Grunenwald, and T. Maillard, Adv. Parallel Comput. 22, 235-242 (2012).

${ }^{12}$ C. G. Durfee and H. M. Milchberg, Phys. Rev. Lett. 71, 2409 (1993).

${ }^{13}$ P. Volfbeyn, E. Esarey, and W. P. Leemans, Phys. Plasmas 6, 2269 (1999).

${ }^{14}$ E. Lifshitz and L. Pitaevski, Physical Kinetics (Butterworth-Heinemann, Oxford, 1981), p. 199.

${ }^{15}$ A. Y. Polishchuk and J. Meyer-Ter-Vehn, Phys. Rev. E 49, 663 (1994).

${ }^{16}$ N. David, D. J. Spence, and S. M. Hooker, Phys. Rev. E 70, 056411 (2004).

${ }^{17}$ G. A. Bagdasarov, P. V. Sasorov, V. A. Gasilov, A. S. Boldarev, O. G. Olkhovskaya, C. Benedetti, S. S. Bulanov, A. Gonsalves, H.-S. Mao, C. B. Schroeder, J. van Tilborg, E. Esarey, W. P. Leemans, T. Levato, D. Margarone, and G. Korn, Phys. Plasmas 24, 083109 (2017).

${ }^{18}$ N. Bobrova and P. Sasorov, Plasma Phys. Rep. 19, 409 (1993).

${ }^{19}$ S. Braginskii, "Transport processes in a plasma," in Reviews of Plasma Physics (Consultants Bureau, 1965), Vol. 1, p. 205.

${ }^{20}$ J. van Tilborg, J. Daniels, A. J. Gonsalves, C. B. Schroeder, E. Esarey, and W. P. Leemans, Phys. Rev. E 89, 063103 (2014).

${ }^{21}$ J. Daniels, J. van Tilborg, A. J. Gonsalves, C. B. Schroeder, C. Benedetti, E. Esarey, and W. P. Leemans, Phys. Plasmas 22, 073112 (2015).

${ }^{22}$ M. Takeda, H. Ina, and S. Kobayashi, J. Opt. Soc. Am. 72, 156 (1982).

${ }^{23}$ P. Sprangle, J. Krall, and E. Esarey, Phys. Rev. Lett. 73, 3544 (1994).

${ }^{24}$ A. J. Gonsalves, K. Nakamura, C. Lin, J. Osterhoff, S. Shiraishi, C. B. Schroeder, C. G. R. Geddes, C. Tóth, E. Esarey, and W. P. Leemans, Phys. Plasmas 17, 056706 (2010).

${ }^{25}$ C. Benedetti, C. B. Schroeder, C. G. R. Geddes, E. Esarey, and W. P. Leemans, Plasma Phys. Controlled Fusion 60, 014002 (2017).

${ }^{26}$ C. Benedetti, C. B. Schroeder, E. Esarey, C. G. R. Geddes, and W. P. Leemans, AIP Conf. Proc. 1299, 250 (2010)

${ }^{27}$ C. B. Schroeder, C. Benedetti, E. Esarey, J. van Tilborg, and W. P. Leemans, Phys. Plasmas 18, 083103 (2011).

${ }^{28}$ C. Benedetti, C. Schroeder, T. Mehrling, B. Djordjevic, S. Bulanov, C. Geddes, E. Esarey, and W. Leemans, in Proceedings of the 2018 IEEE Advanced Accelerator Concepts Workshop (AAC) (IEEE, 2018).

${ }^{29}$ C. Benedetti, C. B. Schroeder, E. Esarey, and W. P. Leemans, Phys. Plasmas 19, 053101 (2012)

${ }^{30}$ J. van Tilborg, A. J. Gonsalves, E. H. Esarey, C. B. Schroeder, and W. P. Leemans, Opt. Lett. 43, 2776 (2018).

${ }^{31}$ J. Van Tilborg, A. J. Gonsalves, E. Esarey, C. B. Schroeder, and W. P. Leemans, Phys. Plasmas 26, 023106 (2019). 